\title{
Massagem cardíaca interna em cães: proposição de nova técnica para pericardiotomia de emergência - tração ligamentar ${ }^{1}$
}

\author{
Internal cardiac massage in dogs: a new technique proposition for emergency \\ pericardiotomy - ligament traction
}

\begin{abstract}
Eduardo Santiago Ventura de Aguiar², Alceu Gaspar Raiser ${ }^{3}$, João Eduardo Wallau Schossler ${ }^{4}$, Ana Néri Christo de Oliveira $^{5}$, Marcelo Weiss ${ }^{5}$, Diego Goulart Sampaio ${ }^{5}$, Juliana Pigatto ${ }^{5}$, Gustavo Demori ${ }^{5}$, André Silva Caríssimi ${ }^{6}$
\end{abstract}

1. Trabalho realizado no Laboratório de Cirurgia Experimental do Programa de Pós-Graduação em Medicina Veterinária da Universidade Federal de Santa Maria

2. Mestre em Cirurgia Experimental pelo Programa de Pós-graduação em Medicina Veterinária da Universidade Federal de Santa Maria

3. Doutor em Cirurgia Experimental pelo Programa de Pós-graduação em Medicina Veterinária da Universidade Federal de Santa Maria

4. Doutor em Cirurgia Experimental pela Escola Paulista de Medicina; sócio da SOBRADIPEC

5. Acadêmico do Curso de Medicina Veterinária da Universidade Federal de Santa Maria

6. Doutor em Animais de Laboratório pela Universidade de São Paulo

\begin{abstract}
RESUMO
Objetivo: Descrever uma técnica de pericardiotomia de emergência, denominada Tração Ligamentar (TL), para diminuir o tempo necessário ao início da Massagem Cardíaca Interna (MCI). Para a MCI necessita-se de toracotomia de emergência e pericardiotomia, ambas em tempo mínimo. A técnica comumente empregada corresponde a pericardiotomia em “T”, cuja execução depende da apreensão do pericárdio com uma pinça de Allis. Este pinçamento é difícil, dificultando a reanimação do paciente. Métodos: Utilizou-se 20 cadáveres de cães, divididos em dois grupos de animais, sendo o Grupo I pericardiotomia em “T” ( $n=10)$ e Grupo II - técnica proposta $(n=10)$. A técnica de TL consistiu na tração do ligamento frenicopericárdico e da secção do pericárdio próximo ao seu ápice. A incisão foi alongada pelos dedos enquanto eram nela introduzidos e permitiu, também, o correto posicionamento do coração na mão do operador, bem como o pronto início da MCI. Resultados: O Grupo I apresentou tempo de execução de 21,79 \pm 0,88 segundo, e o Grupo II de 8,58 \pm 1,38 segundo, sendo $\mathrm{p}<0,0001$, (altamente significativo). Conclusão: A técnica de pericardiotomia por TL impede um tempo maior de isquemia cerebral, por iniciar prematuramente a circulação sangüínea, contribuindo para a sobrevida.
\end{abstract}

Descritores: Parada cardiorrespiratória. Ressuscitação cardiopulmonar. Pericárdio.

\begin{abstract}
Purpose: Describe a technique of emergency pericardiotomy, named as Ligament Traction (LT), to reduce the necessary time to begin the Internal Cardiac Massage. To perform the ICM an emergency toracotomy and pericardiotomy are necessary, both in remote time. The technique usually employed is the " $T$ " pericardiotomy, whose execution depends on the apprehension of the pericardium with an Allis forceps. This apprehension is difficult and complicates the reanimation of the patient. Methods: Twenty canine corpses were divided into two groups: Group I - "T" pericardiotomy (n=10), and Group II - the LT technique ( $\mathrm{n}=10)$. The LT consisted on the traction of the pericardiumphrenic ligament and the section of the pericardium next to its apex. The incision was elongated with the introduction of the fingers, also allowing the positioning of the heart in the hand of the operator and the immediate beginning of the ICM. Results: Group I presented an execution period of $21.79 \pm 0.88$ second, and Group II of $8.58 \pm 1.38$, with $\mathrm{p}<0.0001$ (highly expressive). Conclusion: The technique of pericardiotomy by Ligament Traction concur to outliving, because it avoids a larger time of cerebral ischemia, due to the early beginning of the circulation.
\end{abstract}

Key words: Cardiopulmonary arrest. Cardiopulmonary Resuscitation. Pericardium.

\section{Introdução}

A massagem cardíaca interna foi descrita pela primeira vez em $1874^{1}$, e foi substituída pela massagem cardíaca externa (MCE) em $1960^{2}$. Contudo, em vista dos efeitos lesivos que incorrem do emprego da MCE, a MCI voltou a ser empregada da mesma forma que em situações onde a eficácia da MCE é baixa, como o tórax flutuante, aonde existe, ainda, a possibilidade de lesões internas ${ }^{3,4,5,6}$. Exemplo disso foi o relato de caso de uma paciente que foi ressuscitada com sucesso por meio de MCE, mas que veio a óbito em função das fraturas de costelas e esterno que perfuraram o 
ventrículo e pulmão direitos, levando a paciente ao óbito por choque hemorrágico ${ }^{7}$. Desta forma, é contra-indicada a MCE em pacientes acometidos de tórax flutuante ${ }^{4}$. É citado o relato de uma complicação incomum da MCE, constando de lesão ao sistema venoso de baixa pressão, originando hemorragia venosa e hematoma retrobulbar ${ }^{8}$. Não somente lesões às vísceras internas depõem contra a MCE. Recentes estudos têm demonstrado a superioridade hemodinâmica e de sobrevivência da MCI, relatando melhores níveis de débito cardíaco, fluxo sangüíneo coronariano,carotídeo e cerebral, aumentando, conseqüentemente, o índice de sucesso da ressuscitação ${ }^{9,10,11}$. Isto acontece quando a MCI é aplicada imediatamente em paradas cardíacas, produzindo bons resultados clínicos em seres humanos ${ }^{12}$.

Para a realização da MCI é necessária toracotomia no $5^{\circ}$ espaço intercostal esquerdo, seguida de pericardiotomia num tempo máximo de 30 segundos $^{13}$. Indica-se a apreensão do saco pericárdico com pinças teciduais de Allis para que se possa realizar a pericardiotomia em “T” com uma tesoura de Metzembaum ${ }^{14,15}$. A barra horizontal do "T" deve ser paralela e ventral ao nervo frênico, e a barra vertical compreende uma linha imaginária que vai do ponto médio da cintura cardíaca até o ápice do órgão. A incisão vertical mediana do pericárdio também foi sugerida como meio para pericardiotomia de emergência ${ }^{16}$. Contudo, estes procedimentos são facilmente realizados apenas na presença de efusão pericárdica, pois no coração sem tal afecção, o saco pericárdico é intimamente relacionado ao epicárdio. Neste caso, a sua apreensão torna-se bastante difícil, uma vez que o pericárdio tende a escorregar sob os dentes da pinça tecidual de Allis. O tempo necessário à realização da pericardiotomia de emergência acaba sendo longo, o oposto do que deveria ocorrer numa situação da parada cardiorespiratória, aonde existe a importância de manobras rápidas, evitando o consumo de tempo ${ }^{4}$.

A utilização do aparelho para massagem cardíaca interna minimante invasiva é descrita em humanos, suínos e cadáveres humanos, sendo descritos como de fácil e rápida aplicação, sendo a sua principal vantagem a compressão cardíaca direta sem a necessidade de uma toracotomia ${ }^{17,18 \text {, }}$ ${ }^{19,20}$. Contudo, este aparato ainda não foi testado em cães, e sua disponibilidade ainda é restrita.

Os objetivos deste trabalho constam da proposição de uma nova técnica para a realização da pericardiotomia de emergência, e que esta tenha um intervalo de execução menor do que o procedimento de Pericardiotomia em “T”, a fim de diminuir o tempo necessário ao início da MCI.

\section{Métodos}

Para a realização deste experimento, foram utilizados cadáveres caninos provenientes dos setores de Patologia Veterinária da Universidade Federal do Rio Grande do Sul e da Universidade Federal de Santa Maria. Ao todo foram empregados 20 cadáveres, divididos em dois grupos. No Grupo I, dez cadáveres foram submetidos à técnica de pericardiotomia em “T”, e no Grupo II, outros dez cadáveres sofreram a técnica de Pericardiotomia por Tração Ligamentar. Cada cadáver foi posicionado em decúbito lateral direito, e uma toracotomia no $5^{\circ}$ espaço intercostal foi executada. Após isto, um afastador de costelas foi utilizado para aumentar a área de exposição torácica. Neste ponto o cirurgião realizava um dos dois procedimentos, de acordo com o grupo a que o cadáver pertencia. No grupo I Pericardiotomia em "T", o cronômetro era disparado quando o cirurgião iniciava as tentativas do pinçamento do saco pericárdico, sendo parado apenas quando o cirurgião já estava com o coração posicionado na mão para a primeira compressão cardíaca direta. No grupo II - Pericardiotomia por Tração Ligamentar, o cronômetro era liberado quando o cirurgião iniciava a introdução do dedo indicador sob o ligamento frenicopericárdico, e era parado quando o coração encontrava-se na mão do cirurgião para a primeira compressão cardíaca direta.

\section{Procedimento estatístico}

Os dados obtidos foram analisados pelo pacote estatístico GraphPad InStat ${ }^{\circledR}$, por meio do teste de MannWhitney.

\section{Descrição da técnica de pericardiotomia por tração ligamentar}

Após a realização de uma toracotomia de emergência no $5^{\circ}$ espaço intercostal esquerdo, o cirurgião introduz o dedo indicador esquerdo sob o ligamento frenicopericárdico, tracionando-o até a borda da incisão de toracotomia (Figura 1. A). Com esta manobra o ápice do coração estará deslocado em sentido da sua base, deixando o ápice do pericárdio livre. Tal procedimento ocasiona a visibilização nítida do ligamento e do ápice cardíaco (Figura 1. B). Isto permitirá a incisão parcial do saco pericárdico com uma tesoura de Mayo, sem risco de lesar o miocárdio (Figura 1. C). Lesões vasculares são descartadas, pois nesta região o pericárdio é avascularizado ${ }^{21}$. Posicionam-se, então, as extremidades dos dedos polegar, indicador e médio no interior da incisão, ampliando-a com os mesmos (Figura 1. D). Isto fará com que o coração posicione-se naturalmente na palma da mão do cirurgião, que iniciará a MCI (Figura 1. E). 


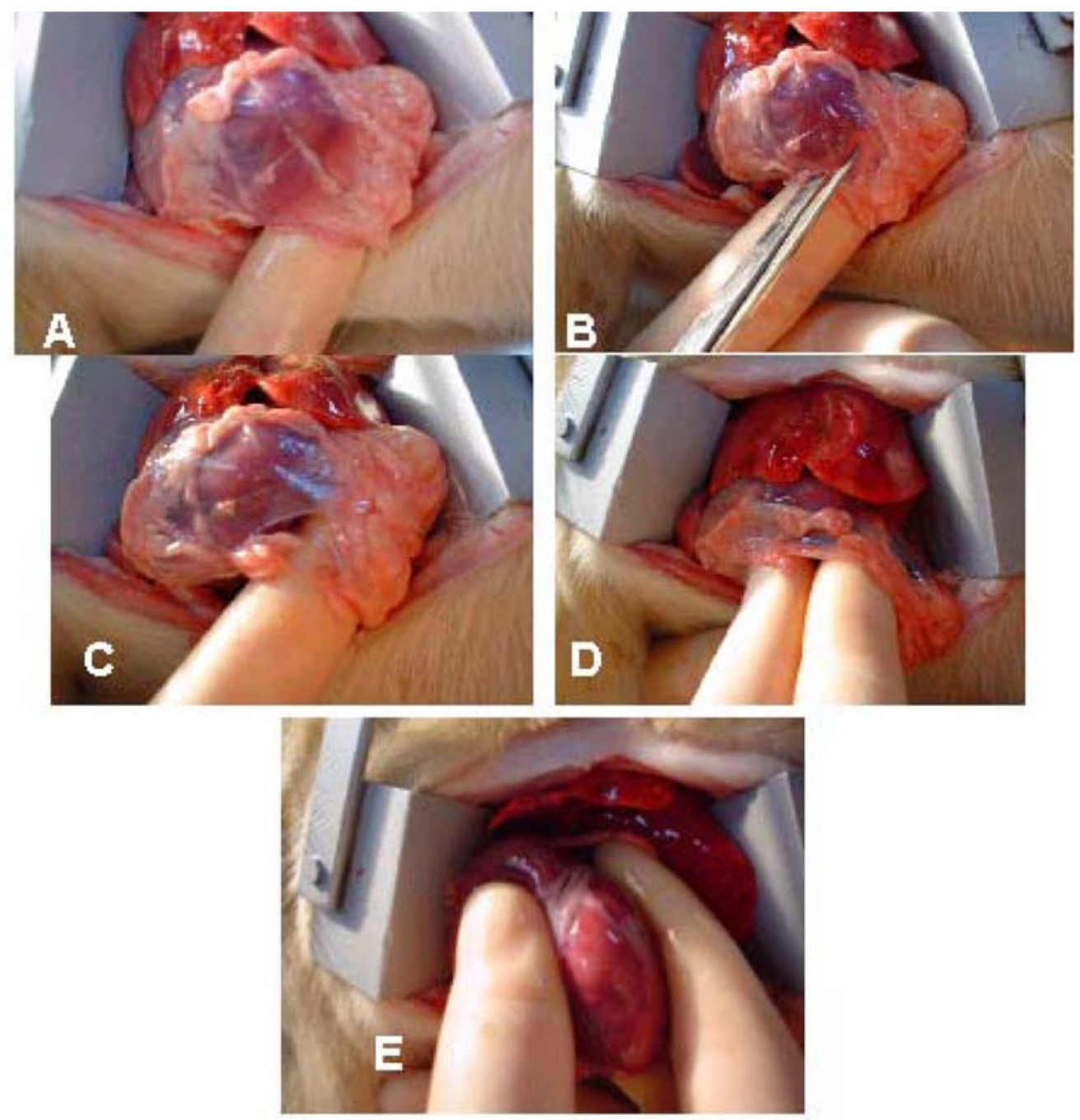

FIGURA 1 - Massagem Cardíaca Interna (MCI) por Tração Ligamentar. O dedo indicador é inserido sob o ligamento frenicopericárdico e é tracionado até a borda da incisão de toracotomia (A). Um ramo de uma tesoura de Mayo é então introduzido sob o ligamento (B), seguido da sua incisão, abrindo o saco pericárdico (C). As extremidades dos dedos polegar, indicador e médio são introduzidas na incisão pericárdica, aumentando o seu diâmetro (D), e permitindo o posicionamento do coração na mão do cirurgião para início da MCI.

\section{Resultados}

Os resultados indicaram que no Grupo I pericardiotomia em “T”- a média de tempo de execução foi de 21,79 $\pm 0,88$ segundos. O Grupo II - pericardiotomia por tração ligamentar - teve média de realização de 8,58 $\pm 1,38$ segundo, aonde $\mathrm{p}<0,0001$ (valor exato), considerado altamente significativo. Os resultados de ambos os grupos encontram-se dispostos na Tabela 1, e os dados estatísticos na Tabela 2.
TABELA 1 - Dados obtidos a partir do Grupo I, Pericardiotomia em “T” e II, Tração Ligamentar.

\begin{tabular}{cc|cc}
\hline \multicolumn{2}{c|}{ Pericardiotomia em "T" } & \multicolumn{2}{c}{ Tração Ligamentar } \\
\hline \hline Cadáver & Tempo (s) & Cadáver & Tempo (s) \\
1 & 20.730 & 1 & 9.320 \\
2 & 22.250 & 2 & 7.050 \\
3 & 21.710 & 3 & 6.070 \\
4 & 21.470 & 4 & 10.790 \\
5 & 23.390 & 5 & 7.540 \\
6 & 22.560 & 6 & 9.170 \\
7 & 20.890 & 7 & 8.730 \\
8 & 21.910 & 8 & 8.350 \\
9 & 20.670 & 9 & 9.160 \\
10 & 22.310 & 10 & 9.660 \\
\hline
\end{tabular}


TABELA 2 - Dados de média e desvio-padrão dos Grupos I - Pericardotomia em “T” e II - Tração Ligamentar.

\begin{tabular}{c|c|c}
\hline Grupo & I - Pericardiotomia em "T" & II - Tração Ligamentar \\
\hline $\mathrm{n}$ & 10 & 10 \\
mínimo & 20,670 & 6,070 \\
máximo & 23,390 & 10,790 \\
Desvio-padrão & 0,8785 & 1,378 \\
Média & 21,789 & 8.584 \\
\hline
\end{tabular}

\section{Discussão}

Os resultados indicam que a MCI por Tração Ligamentar possui um tempo de realização bastante reduzido com relação à pericardiotomia em "T". O tempo máximo para se realizar a toracotomia e a pericardiotomia de emergência é de 30 segundos, o que impede quaisquer perdas de tempo que venham a impedir a rápida realização do procedimento ${ }^{4}$, 13, 14, 15. Com um intervalo de tempo médio de execução bastante curto, ao contrário do constatado na técnica da pericardiotomia em "T", a Tração Ligamentar confere um rápido início da MCI. Esta rapidez promoverá o início precoce de débito cardíaco e fluxo sanguíneo carotídeo e cerebral, produzindo-se melhores índices de sucesso da ressuscitação cardio-respiratória9, ${ }^{12}$. A importância destes dados reflete as numerosas descrições da literatura sobre o emprego da MCI em situações clínicas e experimentais ${ }^{3,5,6,}$ 7, 14, 16. Da mesma forma que a MCI com a técnica de Pericardiotomia em "T", os padrões hemodinâmicos são também obtidos com a técnica proposta, pois a maneira de execução da pericardiotomia de emergência não é responsável por alterações nestes parâmetros.

O fato de o modelo experimental consistir de cadáveres em nada prejudicou o experimento, uma vez que os pacientes que necessitam da MCI encontram-se em parada cardiorespiratória. Da mesma forma, a hemorragia no período pósoperatório é descartada, pois o local de secção do saco pericárdico é isento de vasos sanguíneos ${ }^{21}$.

\section{Conclusão}

Os resultados obtidos com a realização deste experimento permitem concluir que a Pericardiotomia por Tração Ligamentar é um procedimento de realização fácil e rápida, cujo intervalo de execução é menor do que o da Pericardiotomia em “ $T$ ”, diminuindo o tempo necessário ao início da MCI. Desta forma, este menor intervalo de tempo poderá contribuir para a sobrevivência do paciente vítima de parada cárdio-respiratória.

\section{Referências}

1. Hake TG Studies on ether and chloroform, from Prof. Schiff"s physiological laboratory. Practitioner. 1874;12: 241-0.

2. Kouwenhoven WB, Jude MD. Closed-chest cardiac massage. J Am Med Assoc. 1960;173: 94-7.

3. Brunette DD, McVaney K. Hipotermic cardiac arrest: an 11 year review of ED management and outcome. Am J Emer Med. 2000;18: 418-2.

4. Kumar SS, Saith V, Chawla R, Sethi AK, Bhattacharya A.
Successful transdiaphagmatic cardiac resuscitation through midline abdominal incision in patient with flail chest. Resuscitation. 2001;50: 239-1.

5. Hass T, Voelckel WG, Wenzel V, Antretter H, Dessl A, Lindner KH. Revisiting the Cardiac Versus Thoracic Pump Mechanism during Cardiopulmonary Resuscitation. Resuscitation. 2002;58: 113-6.

6. Weinberg G, Ripper R, Feinstein DL, Hoffman W. Lipid emulsion infusion rescues dogs from bupivacaineinduced cardiac toxicity. Reg Anesth Pain Med. 2003;28 (suppl 3): 198-2.

7. Sokolove PE, Willis-Shore J, Panacek EA. Exsanguination due to right ventrticular rupture during closed-chest cardiopulmonary resuscitation. J Emerg Med. 2002;23: 161-4.

8. Evans PJ, Jones MK, Tirlapur VG. An Unusual Complication of External Cardiac Massage. Injury. 1985;16: 477.

9. Vallejo-Manzur F, Varon J, Fromm Jr. R, Baskett P. Moritz Schiff and the History of Open-Chest Cardiac Massage. Resuscitation. 2002;53: 3-5.

10. Hachimi-Idrissi S, Leeman J, Hubloue Y, Huyghens L, Corne L. Open Chest Cardiopulmonary Resuscitation in Out-of-Hospital Cardiac Arrest. Resuscitation. 1997;35: 151-6.

11. Paradis NA, Martin GB, Rivers EP. Use of Open Chest Cardiopulmonary resuscitation after failure of Standard Closed Chest CPR: Illustrative Cases. Resuscitation. 1992;24:61-1.

12. Wingfield WE. Segredos em Medicina Veterinária. Porto Alegre: Artes Médicas Sul; 1998.

13. Raiser AG. Patologia Cirúrgica Veterinária. Santa Maria: UFSM; 1998.

14. Bojrab, M.J. Técnicas Atuais em Cirurgia de Pequenos Animais. São Paulo: Roca; 1996.

15. Fossum, T.W. Cirurgia de Pequenos Animais. São Paulo: Roca; 2002.

16. Voiglio EJ, Coats TJ, Baudoin YP, Davies GD, Wilson AW. Thoracotomie transverse de réanimation. Ann Chir. 2003;128: 728-3.

17. Eynon CA. Minimally Invasive Direct Cardiac Massage and Defibrillation. Resuscitation. 2000;47: 325-8.

18. Buckman,Jr. RF, Badellino MM, Eynon CA, Mauro LH, Aldridge SC, Milner RE, Merchant NB, Buckman III RF, Mercer D, Malaspina PJ, Warren R. Open-Chest Cardiac Massage Without Major Thoracotomy: Metabolic Indicators of Coronary and Cerebral Perfusion. Resuscitation. 1997;34: 247-3.

19. Hanouz JL, Thuaudet S, Ramakers M, Bessodes A, Charbonneau P, Gérard JL, Bricard H. Insertion of the Minimally Invasive Direct Cardiac Massage device (MIDCM): Training on Human Cadavers. Resuscitation. 2002;52: 49-3.

20. Paiva EF, Kern KB, Hilwig RW, Scalabrini A, Ewy GA. Minimally Invasive Direct Cardiac Massage Versus Closed-Chest Cardiopulmonary Resuscitation in a Porcine Model of Prolonged Ventricular Fibrillation Cardiac Arrest. Resuscitation. 2000;47: 287-9.

21. Getty, R. Anatomia dos Animais Domésticos. Rio de Janeiro: Guanabara Kogan; 1986. 
Endereço para correspondência

Eduardo Santiago Ventura de Aguiar

Rua Dr. Ney Cabral, 184

91720-490 Porto Alegre - RS

venturapgvet@yahoo.com.br
Conflito de interesse: nenhum Fonte de financiamento: nenhuma

Recebimento: 15/10/2004

Revisão: 10/11/2004

Aprovação: 14/12/2004

\section{Como citar este artigo:}

Aguiar ESV, Raiser AG, Schossler JEW, Oliveira ANC, Weiss M, Sampaio DG, Pigatto J, Demori G, Caríssimi AS. Massagem cardíaca interna em cães: proposição de nova técnica para pericardiotomia de emergência - tração ligamentar. Acta Cir Bras [serial online] 2005 Mar-Abr; 20(2). Disponível em URL: http://www.scielo.br/acb

Figuras coloridas disponíveis em http://www.scielo.br/acb 